\title{
A High Gain and Directivity Bow Tie Antenna Based on Single-Negative Metamaterial
}

\author{
Li Liu ${ }^{1}$, Chengguang Zhang ${ }^{1}$, Yu Liu ${ }^{2}$ Y Yujin Hua ${ }^{2}$ \\ ${ }^{1}$ Key Laboratory of Exploration Technologies for Oil and Gas Resources, Ministry of Education, Yangtze \\ University, Wuhan, China \\ Li Liu(monicaliuli@163.com); Chengguang Zhang (zhangcg@yangtzeu.edu.cn) \\ ${ }^{2}$ School of Geodesy and Geomatics, Wuhan University, Wuhan, China \\ nansesky1016@163.com,huayujin@163.com
}

\begin{abstract}
This paper presents an antenna loaded with metamaterial to enhance its gain and directivity to improve the performance of a ground-penetrating radar system (GPR). We adjusted the parameters of the antenna to change its equivalent capacitance and inductance and applied an operating frequency of 0.5 GHz-1.2 GHz to create an ultra-wideband antenna. On the basis of this operating frequency, we also designed the corresponding negative permittivity and permeability materials to simulate a patch antenna and material in a $1 \mathrm{GHz}$ frequency band. The high gain and directivity of the antenna loaded with single-negative material were verified by simulation. Compared to the original antenna, the maximum gain increased by $7 \mathrm{~dB}$ and its directivity was greatly improved.
\end{abstract}

Index Terms - Metamaterial-based antenna, negative permittivity, negative permeability, gain enhancement.

\section{INTRODUCTION}

Metamaterials are a type of new artificial electromagnetic material. These include left-handed materials [1-2], zero-refraction materials [3-5], single-negative materials, right/left-hand transmission lines, and photonic crystals, among others. These artificial materials are not present in nature. Metamaterials have been widely used in various fields such as various special antenna designs [6-10], optical imaging [11], electromagnetic wave stealth, subwavelength waveguide devices [12], leaky wave elements [13], and many others. The metamaterials loading on the antenna can result in antenna miniaturization, wide passband, high gain, and other functions. The current small antennas based on metamaterials have been extensively studied. Resonant antennas based on a composite right/left-handed (CRLH) or decentralized design include negative- and zero-order resonant antennas 
[14]. Micro-antennas based on metamaterial loading include negative permeability metamaterials [15], high-permeability shells [16], magnetic photonic crystals (MPC) [17], and Ziolkowski's proposed near-field resonant parasitic antennas [18-19]. Meta-resonator antennas [20-21] include those based on split-ring resonators (SRRs) and complementary split-ring resonator antennas (CSRRs). Loaded hyper-surface antennas [22-23] include electromagnetic band gap structured (EBG) and reactive impedance surface (RIS) antennas. These promote the miniaturization of the antenna size, reduce the surface wave interference, and improve the radiation characteristics.

Bow tie antennas have many advantages, including light weight, easy design and fabrication, better symmetry in radiation, planar structure, and compact size among other factors. These solve the problem of antenna practicality of the traditional ground-penetrating radar (GPR), which is low because of its excessive size, expensive cost, and insufficient bandwidth. But issues remain, as the antenna gain is not high and the directionality is inaccurate and affects the GPR performance. This paper assesses the application of electromagnetic metamaterials in the new GPR antennas. These ultra-wideband antennas have an operating frequency of $0.5 \mathrm{GHz}-1.2 \mathrm{GHz}$. Single-negative material with an operating frequency of approximately $1 \mathrm{GHz}$ is loaded into the antennas to improve their directivity and gain.

\section{ANTENNA DESIGN AND METAMATERIALS}

The design of the double bow tie antenna is shown in Fig. 1. The FR-4 substrate ( $\varepsilon_{r}=4, \tan \delta=0.025$ ) has a length of $220 \mathrm{~mm}$, a width of $168 \mathrm{~mm}$, and a thickness of $1.6 \mathrm{~mm}$. We designed the antenna's radiation plate and feeder on both sides of the substrate. The radiation film and feeder are both $0.03 \mathrm{~mm}$ thick copper. As shown in Figure 1, the size of the radiation film is $148 \mathrm{~mm}$ x $64 \mathrm{~mm}$ x $94 \mathrm{~mm}$. The feeder is symmetrical with a three-section impedance transformer. The first section of the impedance transformer has a length of $10 \mathrm{~mm}$ and a width of $2.6 \mathrm{~mm}$, the second section has a length of $36 \mathrm{~mm}$ and a width of $3.5 \mathrm{~mm}$, and the third section has a length of $36 \mathrm{~mm}$ and a width of $4.1 \mathrm{~mm}$. By adjusting the above parameters, the antenna's bandwidth is maximized, and a standard $50 \Omega$ SMA coaxial connector is used for connection. 


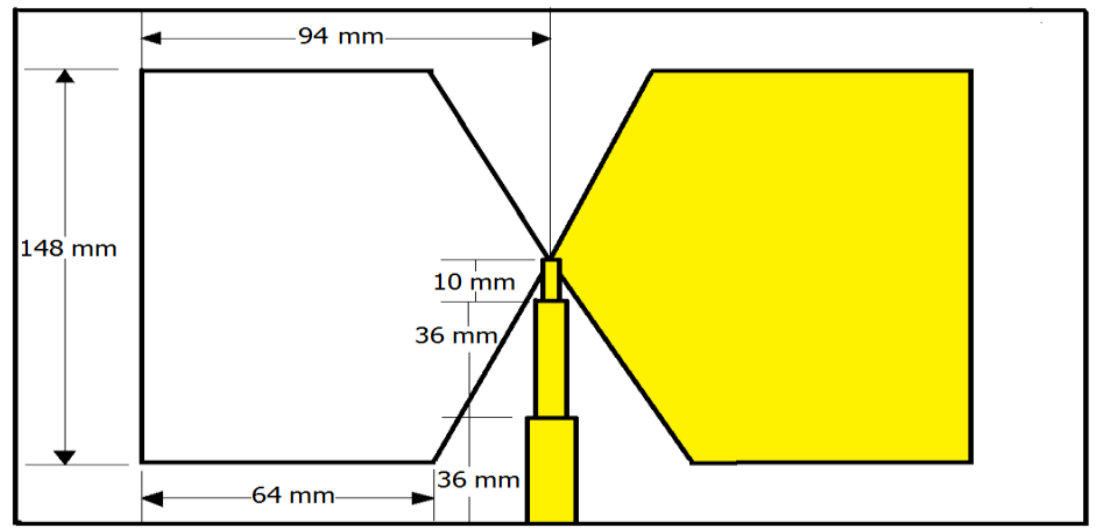

Fig. 1. The structure of a double bow tie antenna.

Fig. 2 shows the antenna simulation. When the $S_{11}$ parameter $<-9 \mathrm{~dB}$ for the passband, the relative bandwidth can reach more than $90 \%$, while the general antenna broadband is only up to $10 \%$. Due to the structure of antenna, because there is no reflector on the back of the radiator plate, the radiation plate capacitance is small. This is conducive to electromagnetic radiation moving outward to avoid the energy that is bounded in the antenna. However, this will also increase the input impedance of the radiation plate, and we found that the angle of the radiation plate is large. According to the equation (1) of the characteristic impedance of a bow tie antenna, a greater flare angle $(\alpha)$ can indirectly reduce the capacitance, thereby increasing the input impedance. In addition, the thickness of the substrate is set at $1.6 \mathrm{~mm}$ compared to the usual $2.5 \mathrm{~mm}$ substrate, so the equivalent capacitance increases. Since the feeding microstrip line is symmetrical, its characteristic impedance will be lower than that of the single-sided microstrip feeder. The input impedance of the antenna is matched as close as possible to $50 \Omega$ by an impedance transformer. The mutual compensation between the equivalent capacitors causes the $S_{11}$ parameter of the antenna to be relatively gentle. In addition, the extension of the bow tie antenna improves the antenna's radiation performance.

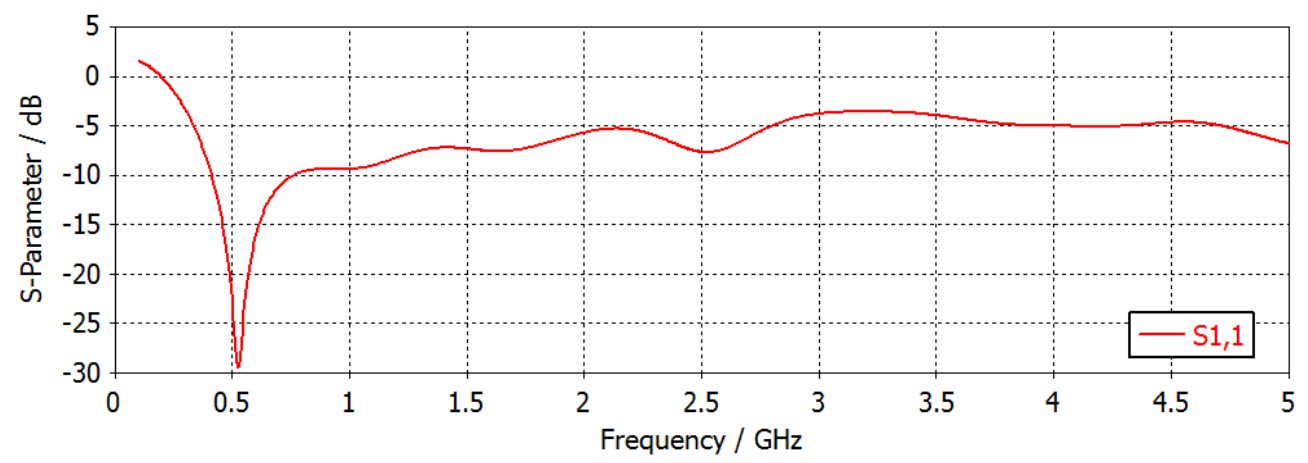

Fig. 2. S-parametric curves of a double bow tie antenna. 


$$
\operatorname{zin}=120 \ln \left[\cot \left(\frac{\alpha}{4}\right)\right]
$$

In a particular frequency, if the permittivity is negative and the permeability is positive, the material is called negative permittivity material. According to an analysis of the constitutive relations, we know that the electromagnetic waves at this time cannot pass through the material. Based on an analysis of the short metal, the structural design of the negative permittivity material is shown in Fig. 3. The FR-4 substrate ( $\varepsilon_{r}=4, \tan \delta=0.025$ ) has a length of $80 \mathrm{~mm}$, a width of $80 \mathrm{~mm}$, and a thickness of $2.5 \mathrm{~mm}$. The yellow surface is pure copper with a thickness of $0.03 \mathrm{~mm}$ and a ring spacing of $2 \mathrm{~mm}$. The back yellow part is $5 \mathrm{~mm}$ in length and $3 \mathrm{~mm}$ in width. For the electromagnetic wave vertical incident material, due to the symmetry of the structure, there is no requirement for the direction of the incident electric field. Fig. 4 shows the S-parameter curve of the single-negative material.

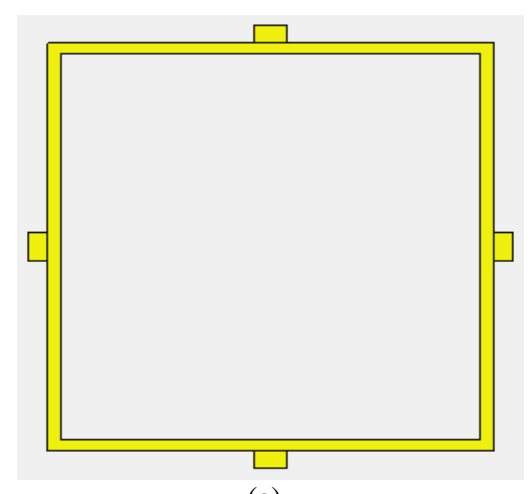

(a)

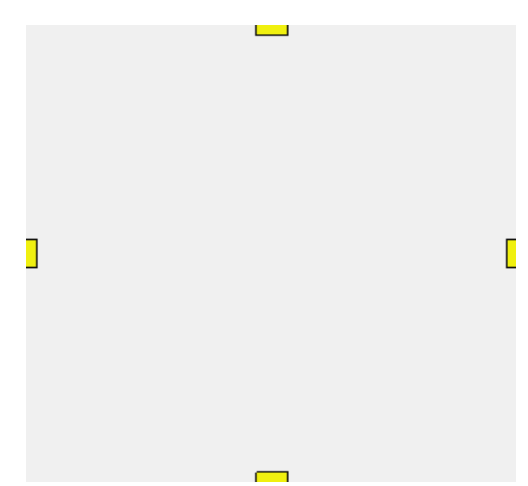

(b)

Fig. 3. (a) (b) show the front and back structure of the materials.

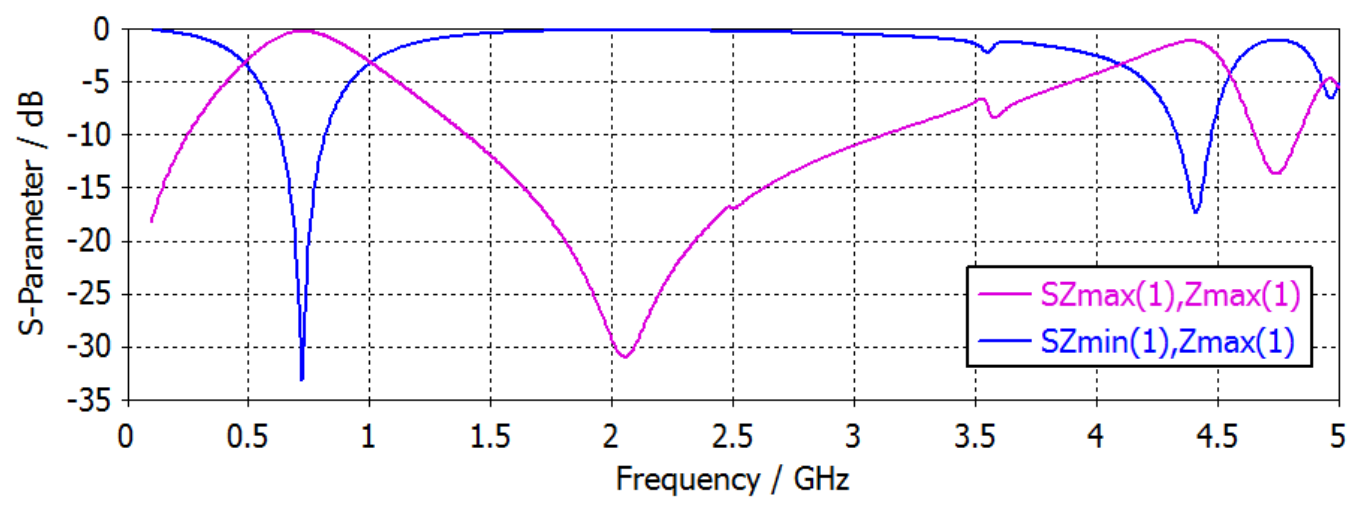

Fig. 4. S-parameter curve of the single-negative material.

Fig. 4 presents the material of the approximately $0.75 \mathrm{GHz}$ stop band. The inversion of its constitutive parameters shows that the permittivity is negative and the permeability is positive in this frequency, which satisfies the negative permittivity required for the experiment. Fig. 5 shows the permittivity and permeability 
of the material changing with the frequency.
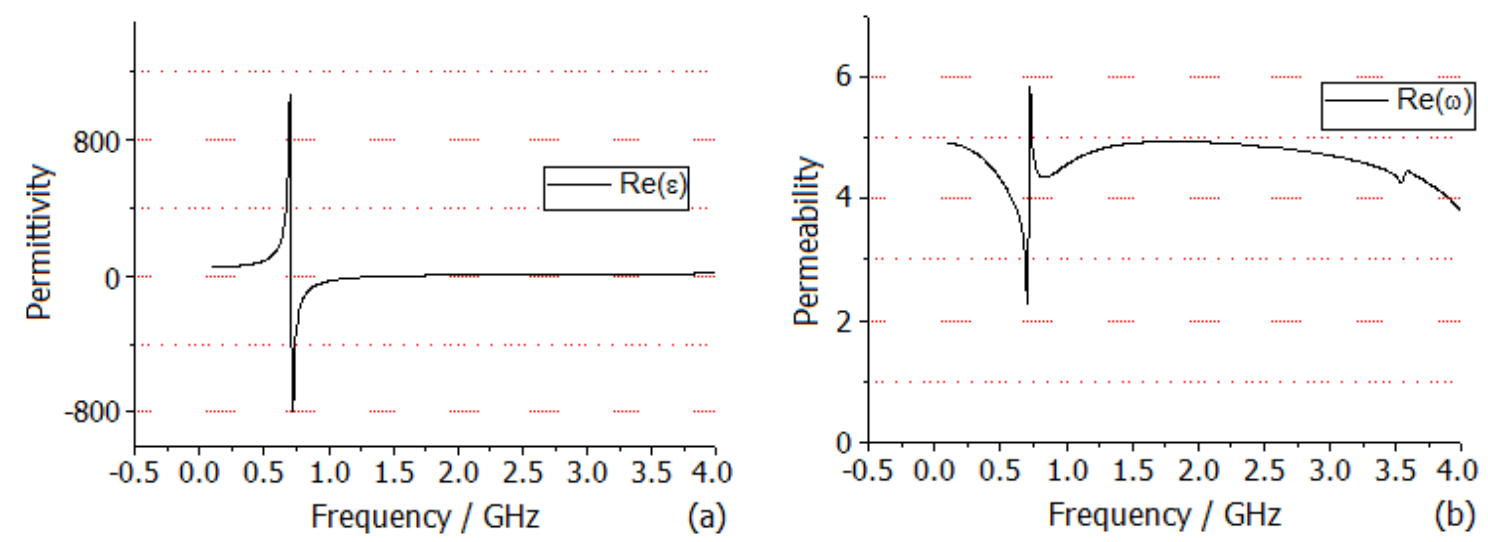

Fig. 5. (a) (b) Permittivity and permeability of the material changing with the frequency.

Material with positive permittivity and negative permeability is called negative permeability material, and we used the electromagnetic vertical incident material. In Fig. 6 , the FR-4 substrate $\left(\varepsilon_{r}=4, \tan \delta=0.025\right)$ has a length of $40 \mathrm{~mm}$, a width of $80 \mathrm{~mm}$, and a thickness of $3 \mathrm{~mm}$. The yellow part is metallic copper symmetrical with the substrate, with a length of $20 \mathrm{~mm}$ and a width of $75 \mathrm{~mm}$. When magnetic resonance occurs, the metal sheet of the substrate on the front and back also acts as an SRR. This material can also engender electrical resonance concomitantly. Fig. 7 shows the equivalent LC circuit of the material when magnetic resonance and electrical resonance occur.

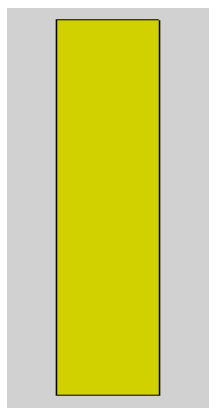

Fig. 6. Material structures for electromagnetic wave vertical incidence. 


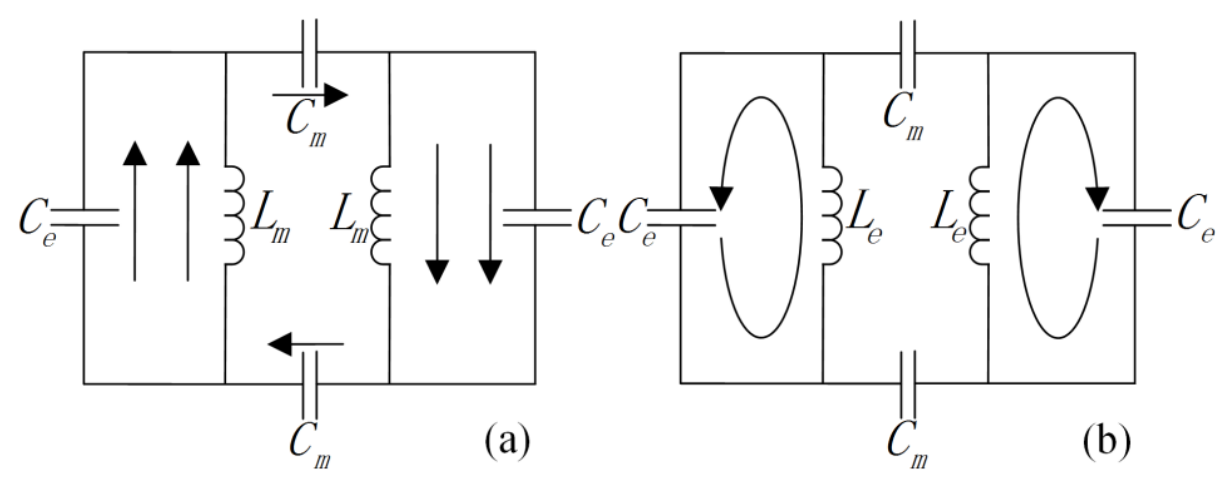

Fig. 7. (a) (b) The equivalent circuit diagram when magnetic resonance and electrical resonance occur

In Fig. 7 (a), Cm, Lm, and Ce are the capacitance between the copper pieces, the inductance of the metal copper sheet, and the capacitance between the adjacent copper pieces, respectively. $\mathrm{Cm}$ and $\mathrm{Ce}$ are the same in Fig. 7 (b) as in Fig. 7 (a), except that the inductance of the metal sheet changes and the calculation is equivalent to the inductance calculation of the double conductor. Fig. 8 shows the S-parameter curves of single-negative material.

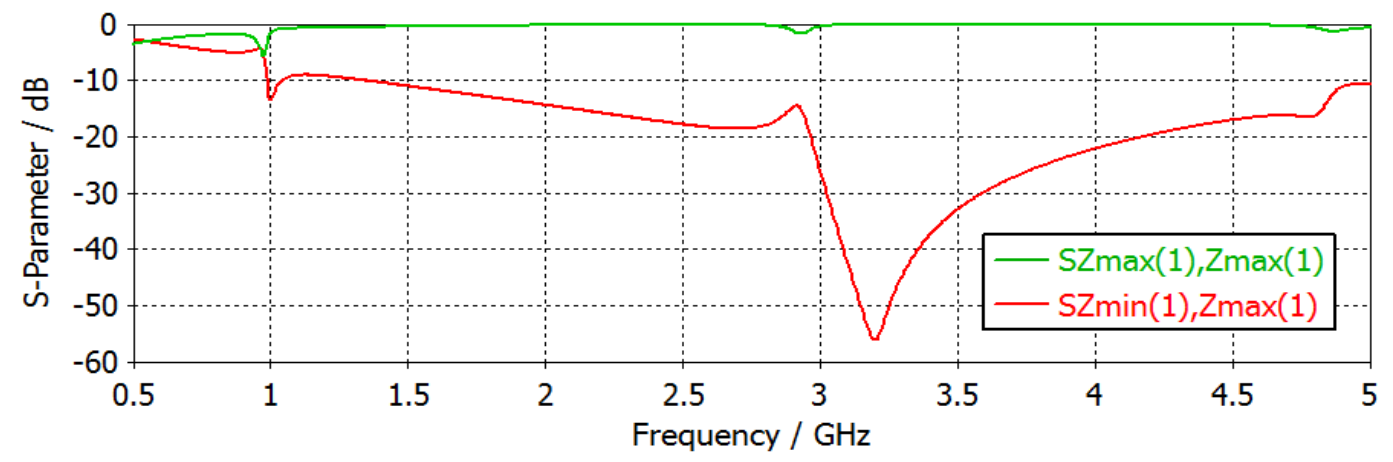

Fig. 8. S-parameter curves of single-negative material.

Through the inversion of the above diagram, we found a frequency of $1 \mathrm{GHz}$ and $3 \mathrm{GHz}$ around the occurrence of magnetic resonance. To verify these results, we observed the surface current at three frequency points, as shown in Fig. 9. 


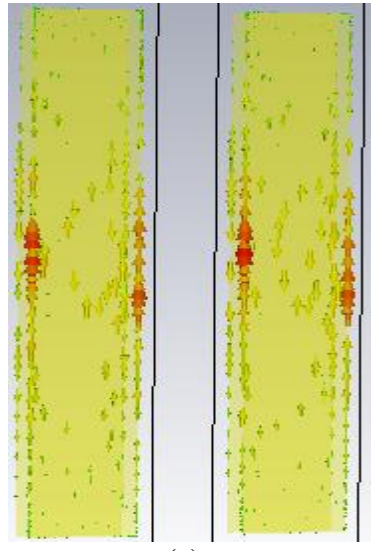

(a)

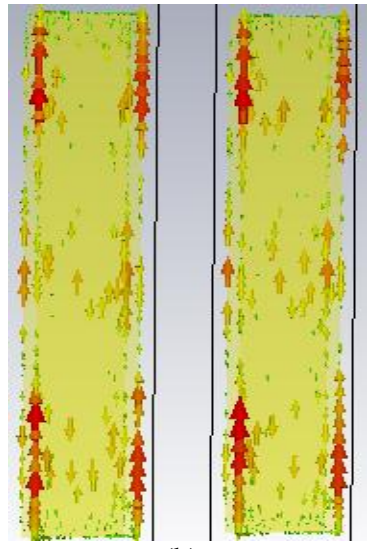

(b)

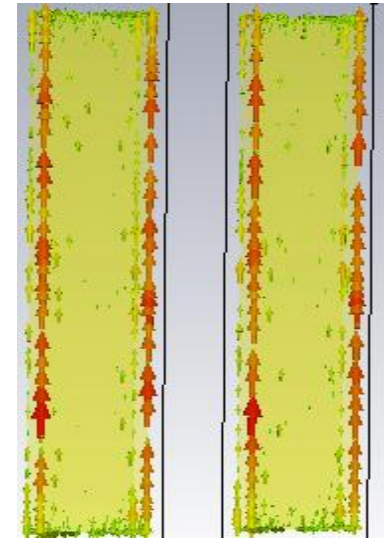

(c)

Fig. 9. Surface flow diagram at (a) $1 \mathrm{GHz}$, (b) $3 \mathrm{GHz}$, and (C) $4 \mathrm{GHz}$.

Fig. 9 (a) shows the surface current distribution at $1 \mathrm{GHz}$. The current direction of the two pieces of metal plate in the front and back in the opposite direction is equivalent to a magnetic dipole, which verifies that the magnetic resonance occurs at $1 \mathrm{GHz}$. Fig. 9 (b) shows the surface current distribution at $3 \mathrm{GHz}$. The metal plate in the front and back on the opposite current flow, and then the current flows to form a clear three circulation. This shows that the magnetic resonance at this time is much stronger than at $1 \mathrm{GHz}$, and the S-parameter values are consistent. Fig. 9 (c) features the metal sheet current direction at 4 GHz. The direction of the current on both sides of the figure is in the same direction, which is precisely the performance of electrical resonance. By comparing the above with the equivalent circuit diagram, we can see that the simulated current direction is consistent with the red current direction in the equivalent circuit, which is further proof of the above. Fig. 10 shows the permittivity and permeability of the material changing with the frequency.
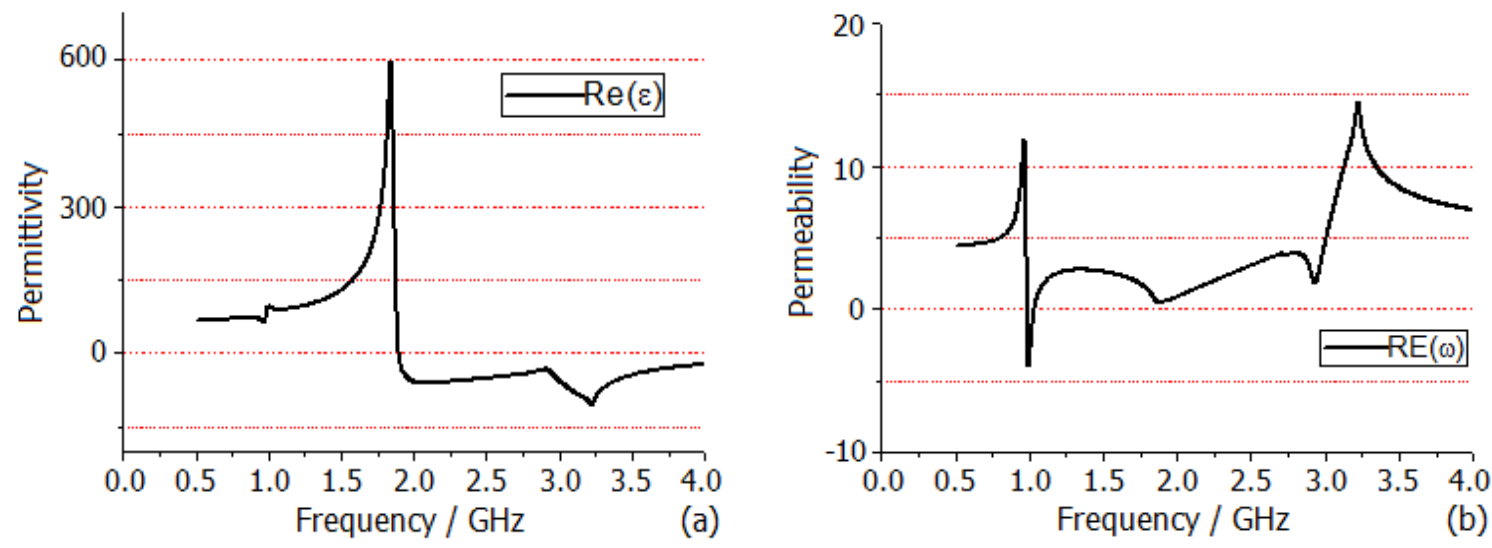

Fig. 10. (a) (b) Permittivity and permeability of the material changing with the frequency.

We have fully verified the above inference through the curve. It is interesting to note that although the 
magnetic resonance is generated at $3 \mathrm{GHz}$ in the surface current diagram, the permeability is not less than zero at $3 \mathrm{GHz}$, which is mainly due to the magnetic dipole formed by the surface current.

\section{SIMULATION AND ANALYSIS OF THE METAMATERIAL-LOADED BOW-TIE}

The upper part of Fig. 11 is the discrete feed antenna and the bottom orange part is the material. The distance between the material and the antenna is $\mathrm{H}, \mathrm{H}=50 \mathrm{~mm}$. Due to the symmetry of the negative permittivity material, there is no requirement for the polarization direction of the radiation field of the antenna in the space during the loading process.

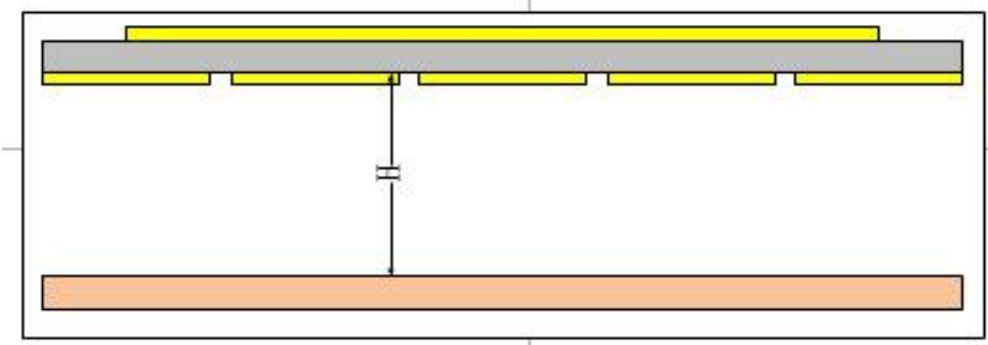

Fig. 11. Antenna structure with loading materials.

The result of comparing the antenna loaded the negative permittivity material and the original antenna is shown in Fig. 12 and Fig. 13.

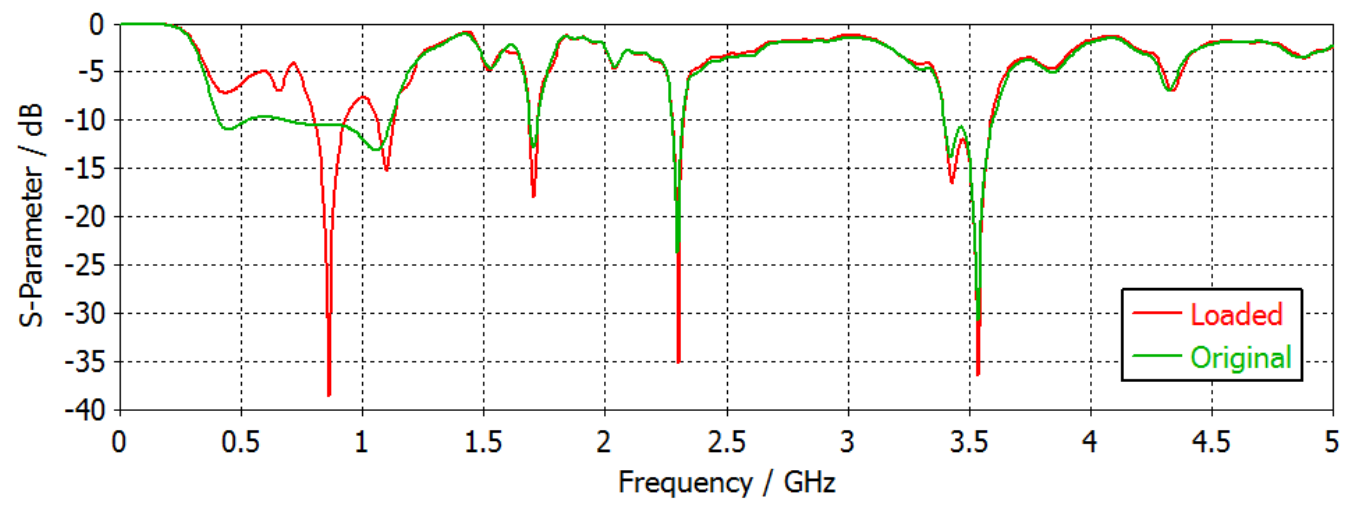

Fig. 12. The S-parameter curves of the antenna with loading negative permittivity material and without loading. 
Farfield Gain Abs (Phi=90)

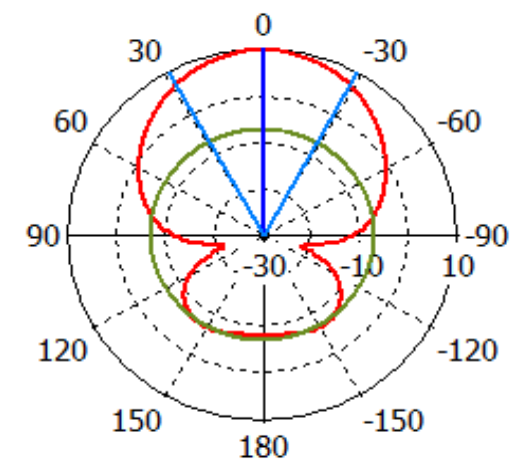

Theta / Degree vs. dB (a)
Farfield Gain Abs (Phi=90)

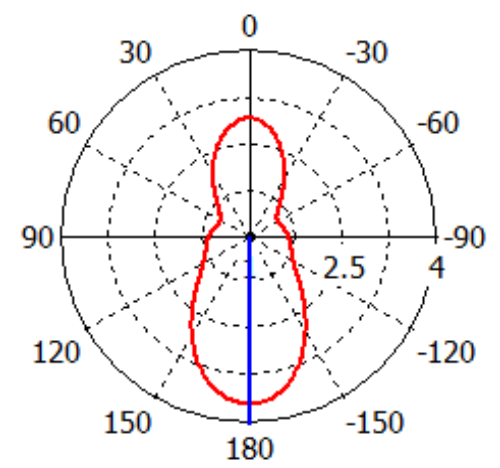

Theta / Degree vs. dB (b)

Fig. 13. (a) (b) The far field direction of antenna (a) with loading negative permittivity material and (b) without loading at $0.86 \mathrm{GHz}$.

In Fig. 12, the red curve is an antenna simulation that loads metamaterial and the green curve is an antenna simulation that does not load metamaterial. We can see the effect of the negative permittivity of the metamaterial on the antenna. It limits the passband of the antenna's $S_{11}$ parameter. Electromagnetic waves propagating to the antenna are reflected back in its negative permittivity frequency range. This is equivalent to the realization of the two radiations, thereby enhancing the gain and directionality of the main radiation direction and inhibiting the antenna's backward radiation. Fig. 13 shows that the gain of the original antenna has been improved. With the highest gain at $0^{\circ}$ in Fig. 13 (a), the main direction of propagation has also shifted from the original backward direction to the forward direction.

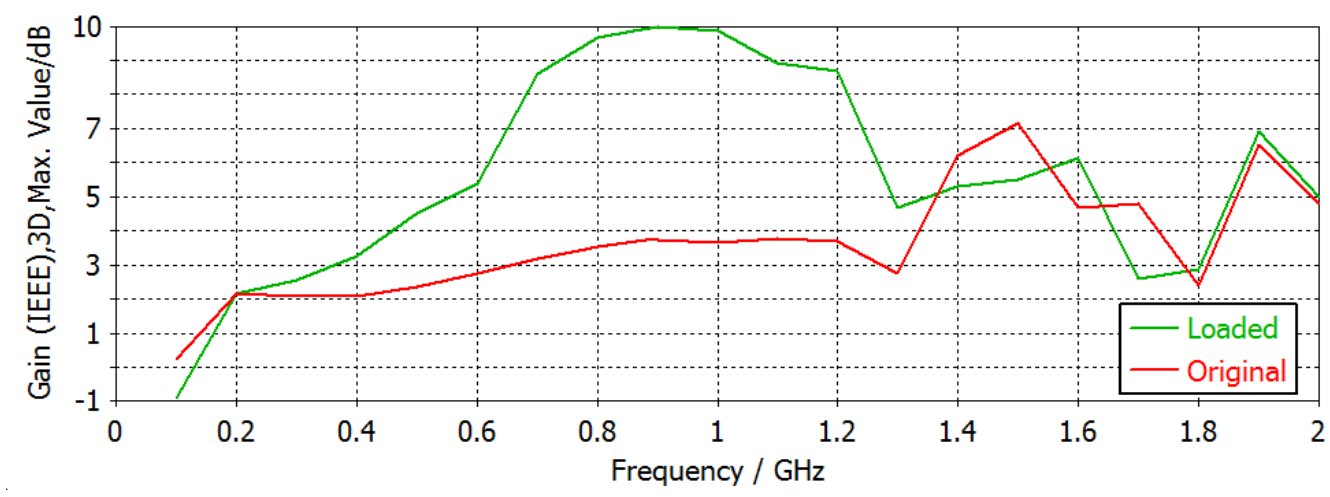

Fig. 14. Gain simulation results of the antenna with loading negative permittivity material and without loading.

Fig. 14 shows the gain for the antenna in two different situations, the green gain for the loaded metamaterial and the red gain for the non-loaded metamaterial. We calculated the antenna gain using CST Microwave Studio, and every $0.1 \mathrm{GHz}$ took a calculation point in the $0.1 \mathrm{GHz}$ to $2 \mathrm{GHz}$ 
frequency range. From the diagram analysis, we can see that the antenna gain without negative permittivity was very gentle, approximately $3 \mathrm{~dB}$ in the frequency range of $0.2 \mathrm{GHz}-1.3 \mathrm{GHz}$. However, the antenna gain with the negative permittivity material was greatly improved, with a maximum increase of $7 \mathrm{~dB}$ in the same frequency range. Therefore, we concluded that the loading of the negative permittivity material enabled the antenna to achieve high gain and high directivity.

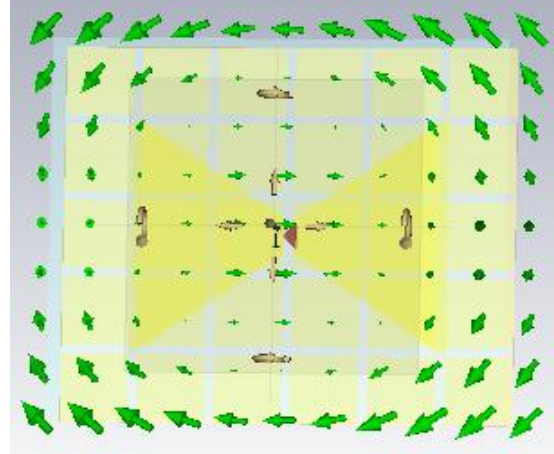

(a)

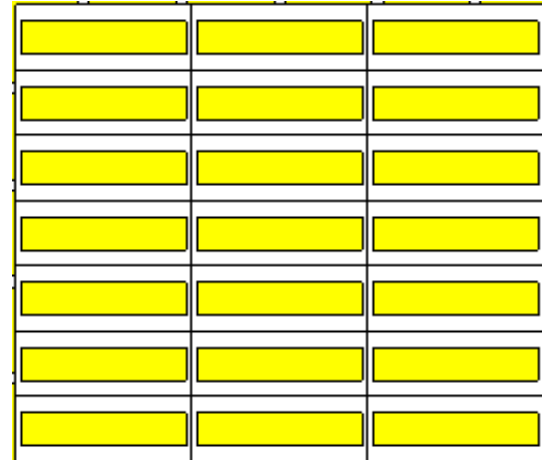

(b)

Fig. 15. (a) Electric field direction $50 \mathrm{~mm}$ from the antenna, (b) the back of the antenna structure with loading material

The negative permeability material we use is the structure of in Fig. 5 (b). Unlike loading negative permittivity materials, we need to know the polarization direction of the radiated electric field of the antenna so the electromagnetic field can generate resonance with the material. Fig. 15 (a) shows the electric field direction $50 \mathrm{~mm}$ from the antenna; (b) shows the back of the antenna with negative permeability material added. The result of comparing the antenna with loaded the negative permeability material and the original antenna is shown in Fig. 16 and Fig. 17.

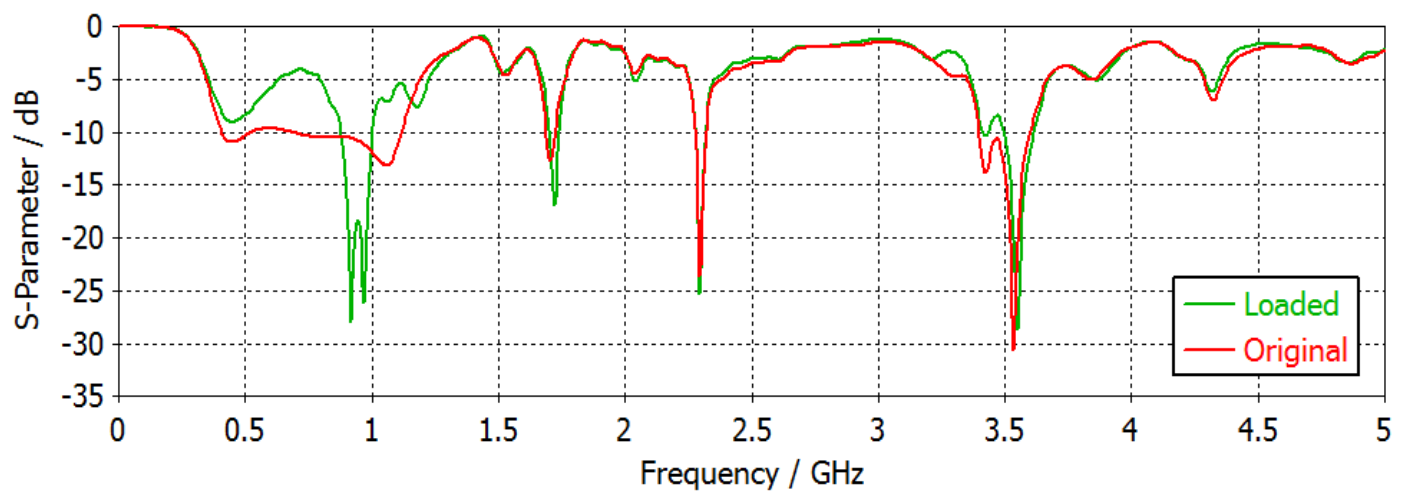

Figure 16. The S-parameters curve of the antenna with loading negative permeability material and without loading 
Farfield Gain Abs (Phi=90)

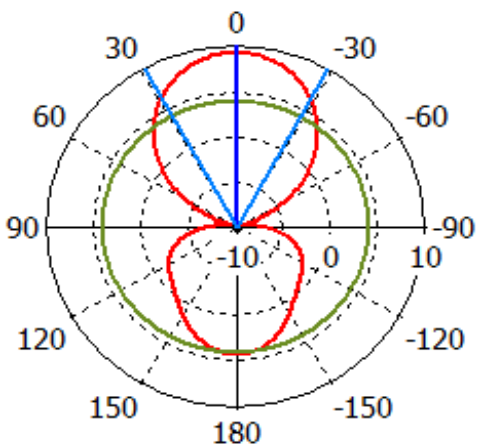

Theta / Degree vs. dB (a)
Farfield Gain Abs $($ Phi $=90)$

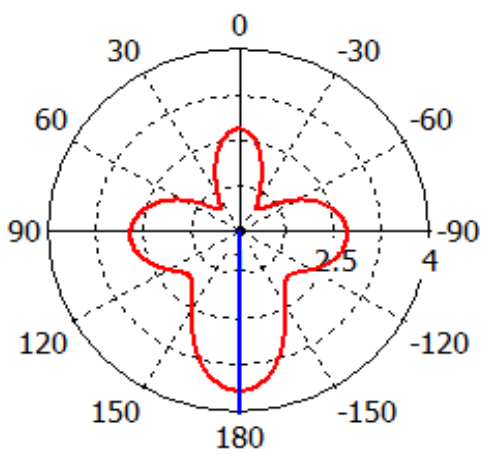

Theta / Degree vs. dB (b)

Figure 17. The far field direction of the antenna (a) with loading negative permeability material and (b) without loading at 1

$\mathrm{GHz}$

In Fig. 16, the green curve is an antenna simulation that loads metamaterial and the red curve is an antenna simulation that does not load metamaterial. We can see the effect of the negative permeability of the metamaterial on the antenna. It also limits the passband of the antenna's $S_{11}$ parameter. Electromagnetic waves propagating to the antenna are reflected back in its negative permeability frequency range. This is equivalent to the realization of the two radiations, thereby enhancing the gain and directionality of the main radiation direction and inhibiting the antenna's backward radiation. Fig. 17 shows that the gain of the original antenna has improved. With the highest gain at $0^{\circ}$ in Fig. 17 (a), the main direction of propagation has also shifted from the original backward direction to the forward direction.

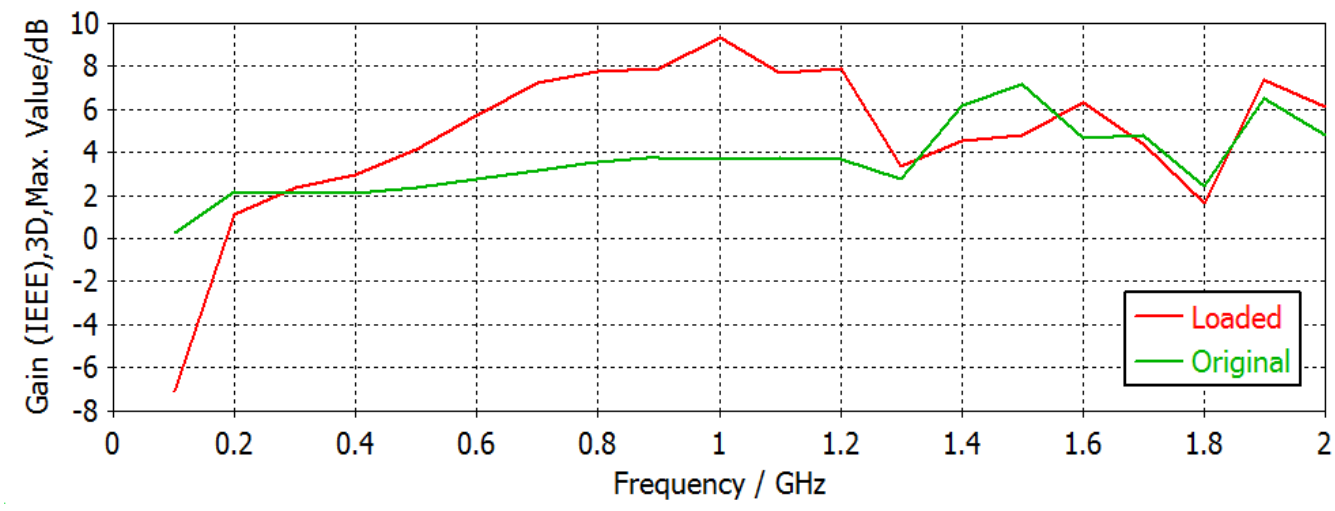

Fig. 18. Gain simulation results of the antenna with loading negative permeability material and without loading

Fig. 18 shows the gain for the antenna in two situations, the red gain for the loaded metamaterial and the green gain for the non-loaded metamaterial. We calculated the antenna gain using CST 
Microwave Studio, and every $0.1 \mathrm{GHz}$ took a calculation point in the $0.1 \mathrm{GHz}$ to $2 \mathrm{GHz}$ frequency range. From the diagram analysis, we can see that the antenna gain without negative permeability is very gentle, approximately $3 \mathrm{~dB}$ in the frequency range of $0.2 \mathrm{GHz}-1.3 \mathrm{GHz}$. However, the antenna gain with the negative permittivity material was greatly improved, with a maximum increase of $6 \mathrm{~dB}$ in the same frequency range. Therefore, we concluded that the loading of the negative permeability material enabled the antenna to achieve high gain and directivity.

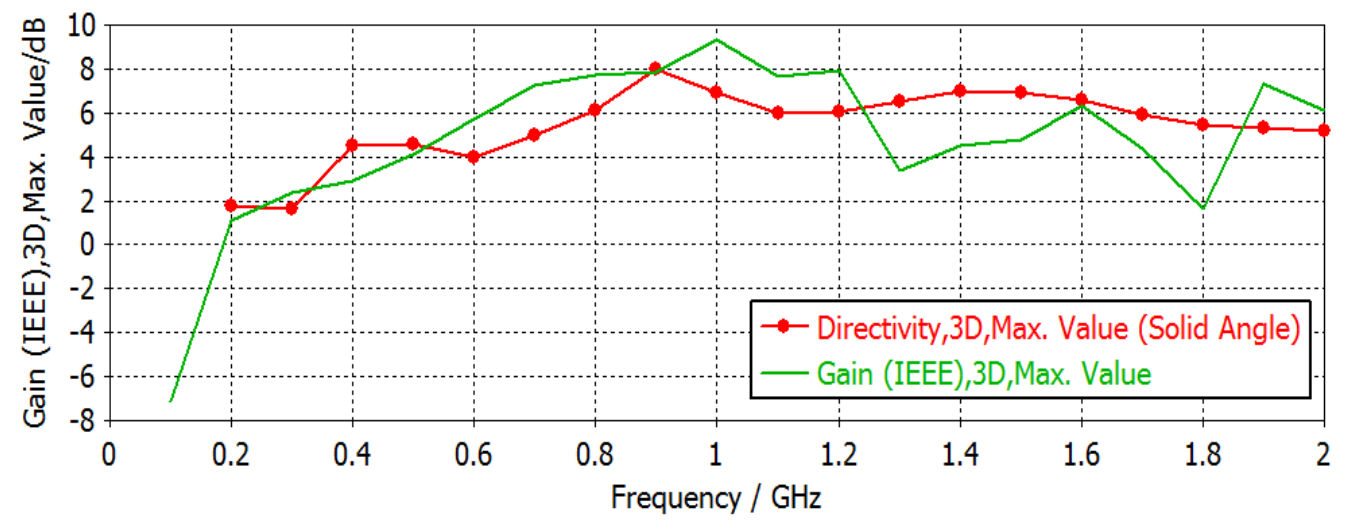

Fig. 19. The maximum gain comparison

We compared the antenna of this article with the antenna proposed by Rashmiranjan Nayak et al[24]. Fig. 19 shows the maximum gain of the two antennas (the red line indicates the gain of antenna designed by Rashmiranjan Nayak, and the green line is the antenna gain in this article). It can be seen that in the operating frequency range from $0.5 \mathrm{GHz}$ to $1.2 \mathrm{GHz}$, our antenna gain has increased about $2 \mathrm{~dB}$, while the size of the antenna designed by Rashmiranjan Nayak is about twice as large as our antenna.

\section{CONCLUSION}

In summary, the paper presented a high gain and directivity bow tie antenna of a GPR system with metamaterial. An ultra-wideband antenna with an operating frequency of $0.5 \mathrm{GHz}-1.2 \mathrm{GHz}$ was designed using CST Microwave Studio; the negative permittivity and permeability material had a working frequency of approximately $1 \mathrm{GHz}$, realizing the design and simulation of a patch antenna and material in the $1 \mathrm{GHz}$ frequency band. Through the S-parameter inversion of the design material and the analysis of the surface current at the corresponding frequency, we verified the material's characteristics with equivalent negative constitutive parameters. The high gain and directivity of the 
antenna loaded with the single-negative material were verified via simulation. Compared to the original antenna, the maximum gain increased by $7 \mathrm{~dB}$ and its directivity was greatly improved. Consequently, it is effective and feasible to load a single-negative metamaterial onto an antenna for high gain and directivity.

\section{ACKNOWLEDGMENT}

This work was supported by the National Natural Science Foundation of China (grant number 41774116).

\section{REFERENCES}

[1] Xiong, H., Hong, J.S., Jin, D.L. and Z. M. Zhang, A Novel Structure for Broadband Left-handed Metamaterial, Chin. Phys. B, 21, pp. 4101-4105, 2012.

[2] Kafesaki, M., Tsiapa, I., Katsarakis, N. \& Koschny, Th., Left-handed Metamaterials: the Fishnet Structure and Its Variations, Physical Review B, 75, pp. 5114-5122, 2007.

[3] Sun, L. \& Yu, K.W., Strategy for Designing Broadband Epsilon-near-zero Metamaterial with Loss Compensation by Gain Media, Applied Physics Letters, 100, pp. 1903-1906, 2012.

[4] Soemphol, C., Sonsilphong, A. \& Wongkasem, N., Metamaterials with Near-zero Refractive Index Produced Using Fishnet Structures, Journal of Optics, 16, pp. 5104-5109, 2014.

[5] Sun, L., Gao, J. \& Yang, X.D., Broadband Epsilon-near-zero Metamaterials with Step-like Metal-dielectric Multilayer Structures, Physical Review B, 87, pp. 5134-5139, 2013.

[6] Nilavalan, R., Hilton, G.S. \& Benjamin, R., Wideband Printed Bowtie Antenna Element Development for Post Reception Synthetic Focusing Surface Penetrating Radar, Electronics Letters, 35, pp. 1771-1772, 1999.

[7] Soh, P.J., Mercuri, M., Pandey, G. \& Vandenbosch, G.A.E., Dual-Band Planar Bowtie Monopole for a Fall-Detection Radar and Telemetry System, IEEE Antennas and Wireless Propagation Letters, 11, pp. 1698-1701, 2012.

[8] Byers, K.J., Harish, A.R., Seguin, S.A. \& Leuschen, C.J., A Modified Wideband Dipole Antenna for an Airborne VHF Ice-Penetrating Radar, IEEE Antennas and Wireless Propagation Letters, 61, pp. 1435-1443, 2012.

[9] Mahmoud, A.M., Hoorfar, A. \& Thajudeen, C., Enhanced Design of Bowtie Antennas over High-Impedance Surfaces: An Evolutionary Approach, IEEE Antenna and Propagation Society Meeting, Jul. pp. 270-271, 2013.

[10] Ranasinghe, H.M.P.B., Senanayake, S.M.P., Senarathne, U.I.P., \& Gunawardena, A.U.A.W., Design of a Low Cost Cavity Backed Wideband Bow-tie Antenna for Ground Penetrating Radar Systems, IEEE 8th International Conference on Industrial and Information Systems, Dce. pp. 370-372, 2013.

[11] Pentry, J.B., Negative Refraction Makes a Perfect Lens, Physical Review Letters, 85, pp. 3966-3969, 2000.

[12] Ozlem, O. \& Mustafa, K., Utilization of Anisotropic Metamaterial Layers in Waveguide Miniaturization and Transitions, IEEE Microwave and Wireless Components Letters, 17, pp. 754-756, 2007.

[13] Dong, Y.D. \& Itoh, T., Composite Right/left-handed Substrate Integrated Waveguide Leaky-wave Structures, IEEE Transactions on Antennas and Propagation, 59, pp. 767-775, 2011.

[14] Paulotto, S., Baccarelli, P., Frezza, F. \& Jackson, D.R., Full-wave modal dispersion analysis and broadside optimization for a class of microstrip CRLH leaky-wave antennas, Microwave Theory and Techniques, IEEE Transactions on, 56,pp. 2826-2837, 2008.

[15] Stuart, H.R. \& Pidwerbetsky, A., Electrically small antenna elements using negative permittivity resonators. Antennas and Propagation, IEEE Transactions on, 54, pp. 1644-1653, 2006.

[16] Stuart, H. R. \& Yaghjian, A.D., Approaching the Lower Bounds on Q for Electrically Small Electric-dipole Antennas Using High Permeability Shells, Antennas and Propagation, IEEE Transactions on, 58, pp. 3865-3872, 2010.

[17] Mumcu, G., Sertel, K. \& Volakis, J.L., Miniature Antenna Using Printed Coupled Lines Emulating Degenerate Band Edge Crystals, Antennas and Propagation, IEEE Transactions on, 57, pp. 1618-1624, 2009.

[18] Ziolkowski, R.W., Jin, P., Nielsen, J. \& Tanielian, M., Experimental Verification of Z Antennas at UHF Frequencies, Antennas and Wireless Propagation Letters, 8, pp. 1329-1333, 2009.

[19] Jin, P. \& Ziolkowski, R.W., Low-Q, Electrically small, Efficient Near-field Resonant Parasitic Antennas, Antennas and Propagation, IEEE Transactions on, 57, pp. 2548-2563, 2009.

[20] Dong, Y., Toyao, H. \& Itoh, T., Design and characterization of miniaturized patch antennas loaded with complementary split-ring resonators, Antennas and Propagation, IEEE Transactions on, 60, pp. 772-785, 2012.

[21] Kim, I. k., \& Varadan, V. V., Electrically Small, Millimeter Wave Dual Band Meta-resonator Antennas, Antennas and Propagation, IEEE Transactions on, 58, pp. 3458-3463, 2010.

[22] Dong, Y., Toyao, H. \& Itoh, T., Miniaturized zeroth order resonance antenna over a reactive impedance surface, International Workshop on Antenna Technology, May. pp. 58-61, 2011. 
Journal of Microwaves, Optoelectronics and Electromagnetic Applications, Vol. 17, No. 2, June 2018 DOI: http://dx.doi.org/10.1590/2179-10742018v17i21116

[23] Zhu, S. \& Langley, R., Dual-band wearable textile antenna on an EBG substrate. Antennas and Propagation, IEEE Transactions on, 57, pp. 926-935, 2009.

[24] Rashmiranjan N., Subrata M., \& Sarat K. P., Design and simulation of compact UWB Bow-tie antenna with reduced end-fire reflections for GPR applications, Wireless Communications Signal Processing and Networking (WiSPNET) International Conference on, pp. 1786-1790, 2016. 